floor, which also coincided with contraction of the external urethral sphincter as shown by cystography during stimulation of the bladder, this could explain the high residual urine.

Ascoli, R., replied that the electrical stimulation was not used in spastic bladdersi.e. in upper neurone lesions. It was used only in flaccid cases-i.e. cauda equina lesions or in lesions where innervation of the abdominal muscles was absent. One knew that the abdominal contraction was very important in the act of micturition, and in cases where the abdominal muscles were present the act of voiding was possible. In other cases the bladder was practically completely isolated and therefore had to be stimulated.

Benassy, $\mathcal{F}$. (France), said he had tried many times to use electronic devices for the bladder. Up to now they were always a failure, but they were continuing to try. They used them mainly in two cases: (a) Retention of the bladder in a case with hyperspasticity. The result was very bad because the spasticity was due to spasticity of the smooth muscles and after electronic stimulations, of course, it was worse. One wanted relaxation rather than stimulation. (b) In a case of low cauda equina, the incontinence was due to paralysis of the flaccid muscles of the pelvis and these flaccid muscles did not respond to electronic stimulation. In this case again they gained nothing.

\title{
A CASE OF TRANSPOSITION OF THE MUSCULO-CUTANEOUS NERVE UPON THE MEDIAN NERVE
}

\author{
By Jean Benassy, M.D. \\ Hospital Raymond-Poincare, Paris Garches, France
}

WE should like to report a case of successful transposition of the musculo-cutaneous nerve upon the median nerve in a tetraplegic patient, which is of interest both in the field of paraplegia as well as of peripheral nerve surgery.

\section{Case History}

A. W., 37 years old, was involved in a motor-car accident on I 2 January I962, and sustained a tetraplegia as a result of a fracture dislocation of the 5 th cervical vertebra upon the 6th.

He was admitted first to a hospital of the nearest town, and on I3 January transferred to a neurosurgical service in Paris. On I5 January 1962, it became necessary to perform a tracheostomy, which was discontinued on 22 February 1962.

The patient was transferred on 28 February 1962 to Garches, where he remained up to March 1963. He did not develop any complications, such as pressure sores, periarticular ossifications or urinary infection, and the rehabilitation of the bladder was good.

Eleven months after the accident, the muscular recovery was as follows:

Right Side

Very good function (5) of what we call the 5th cervical root distribution:

Deltoideus

Supra-spinatus

Infra-spinatus

Biceps

Brachialis

Brachio-radialis

Supinator brevis

Good function (4) in the distribution of C6: 
Pectoralis major (upper head)

Latissimus dorsi

Teres major

Subscapularis

Extensores carpi radiales

Pronator teres

Long head of triceps.

Left Side

Very good function (5) in the distribution of $\mathrm{C}_{5}$.

Poor function in the distribution of C6:

Subscapularis (5)

Extensor carpi radialis longus and brevis (4).

The sensory function was better on the left side than on the right.

We thought that on the left side we could take the risk of sacrificing the distal part of the innervation of the musculo-cutaneous nerve to the brachialis internus and lower head of the biceps to gain some useful function of muscles of the hand innervated by the median nerve, and thought that the re-education could give enough strength to the remaining flexors of the elbow.

The operation was performed on I December 1962. The musculo-cutaneous nerve was cut at mid-height of the arm, and sutured easily and without tension to the median nerve, with the help of magnifying glasses. The anastomosis was reinforced by suture to the brachialis internus nerve using it as a splint.

Six months later, flickers could be observed in the three first fingers. Eight months later, examination showed a recovery of value ' 3 ' of the pronator teres, flexor carpi radialis, and tendons of the index and middle fingers corresponding to the flexor digitorum profundus. A recovery of value ' 2 ' was also found in the flexor pollicis longus.

The sensibility which had been lost after the operation had nearly completely recovered, better for touch than for the appreciation to pain and temperature sensibility.

The patient is now able to eat alone, without any gadget (except for cutting meat); he can type, take a cigarette and light a match, using the reactivated fingers. He can now also use his left arm to drive his wheelchair.

The loss of strength of the elbow-flexors is still marked $(7 \mathrm{~kg}$. against $\mathrm{I} 2$ on the right side), but gives only slight disturbance. In May 1965 a new test was made. No new muscular recovery is to be noted. The functional status is demonstrated by a film.

An electromyographic study was performed in May 1965 by doctor Lacuire and showed a return of electric activity in the four clinically active muscles. Pronation is achieved by the pronator teres and not the pronator quadratus. There exists a synergic action of the three heads of the flexors of the fingers which act together either with the flexion of the wrist or pronation of the forearm.

\section{DISCUSSION}

First it must be stressed that, on the whole, the recovery is rather poor. The patient was aged 37, the operation was performed I I months after the accident. It is known that, except in young patients operated rather soon after the accident, wonderful results are not to be expected in nerve surgery. But we could not take an earlier decision in order to be sure that no spontaneous recovery could take place. Nevertheless the recovery has proved very useful for the patient, who can now type with the left hand, eat nearly alone, light a match and drive his wheelchair.

Unfortunately most patients suffering from tetraplegia after dislocation of this type are not suitable for such a procedure because they do not have the use of the extensors of the wrist, as it was fortunately the case in our patient. 
We should be happy to know if some other operations of this type have already been performed. Let us stress again the fact that technically it is an easy procedure since it is performed upon anatomically normal nerve trunks.

\section{RÉSUMÉ}

Le cas d'une transposition du musculo-cutané sur le médian chex un tétraplégique C5-C6 est rapporté.

Il en est résulté une récupération du rond pronateur, grand palmaire, une récupération partielle du fléchisseur profond (index et médius) et du fléchisseur profond du pouce. aucune récupération thénarienne.

La flexion du coude a été définitivement réduite à la suite de la transplantation.

\section{SUMMARY}

A case of tranposition of the musculo cutaneous nerve on the median nerve in a tetraplegic below $\mathrm{C}_{5}$ C6 is reported, the operation resulting in a partial recovery of the pronator teres, flexor carpi radialis, index and middle fingers of the flexor digitorium profundus and the flexor pollicis longus. No recovery was found in the thenar muscles. Flexion of the forearm was definitely reduced as a result of the nerve transposition.

\section{Discussion}

Michaelis, L. S. (England), thought the transposition of the musculo cutaneous nerve into the median in this particular case a very good idea. It had also been carried out technically well and the result was quite startling. It opened the door to a new approach to surgical attempts at improvement of finger function, and looking at the results of those few tendon procedures or bone grafts which had been used in the past he for one would be very grateful if something better could take their place. It was true that with tendon transfers one could achieve good results in individual cases, but in spite of technical successes of these operations he more and more hesitated to employ this kind of operation. The reason was that, as Dr. Guttmann had repeatedly pointed out, the patient if given time and given a free range of his own energy and practice would acquire in his paralysed upper limb a new kind of co-ordination between joints and those muscles which had survived. He would acquire a technique of using even paralysed fingers to a degree of perfection which one would hardly believe if one did not see it. One must realise that there was a degree of physiological adaptation to the lack of active muscles which surgeons in general should hesitate to disturb. One might gain an individual function-say a pinch - but what was the price one had to pay? The price might be a permanent disturbance of the acquired co-ordination and this price might be too high. With Benassy's approach there was a chance that, every now and then, in one individual patient one would be able to pick out a particular power nerve to implant in a powerless nerve. His only criticism of Benassy's paper was that he had made the same mistake as other surgeons had made before. He had said the operation was easy. It might be easy for him, but there were few surgeons, neurological or orthopaedic, who had sufficient experience of peripheral nerve surgery.

Guttmann, L., asked a question from a physiological point of view. He could not discover any function of the thenar muscles, i.e. abductor pollicis brevis, opponens and lumbricals. If one succeeded in having this function returned, which he still doubted, 
that would indeed be a great practical advance. To have just a finger flexion was a rather minor point and he was glad that Dr. Benassy had not added to the restoration of the weak finger flexion the restoration of the extensor digitorum longus by a tendon transplant. If he had done so this would have resulted in a claw hand and the patient would have been much worse off than before. He also noticed in the film demonstrated by Dr. Benassy that the patient typed with the hand on which nothing had been done, and it had not occurred to him that the finger grip of the recovered flexors differed significantly from that of those patients who flex the fingers passively by the trick movement of the extensor carpi radialis. He would be very glad indeed if Dr. Benassy would follow up that particular case and let them know next year, because the rate of recovery, according to his own experimental studies during the war, took a long time. Moreover, it was considered that the musculo cutaneous nerve was not a very strong nerve to guarantee an outgrowth of fibres in such a great number that the small muscles of the hand, in particular the abduction and opposition of the thumb, could be restored. It would be of great importance to know next year whether some function of the opponens and abductor pollicis brevis had occurred. Another point Dr. Benassy mentioned was that the sensibility in the distribution of the lateral cutaneous nerve of the forearm had recovered. That did not mean anything, as it was well known that by overlap of the median cutaneous and dorsal cutaneous nerves of the forearm, the result was always very good even in the presence of complete interruption of the musculo-cutaneous nerve. He would like to know how far and what type of sensibility had recovered in the distribution of the median nerve: (a) pain, (b) tactile and discriminative sensibility.

Benassy, $\mathcal{F}$., replied that with regard to tactile sensibility - the patient could recognise things as usual; for pain, nearly nothing, for hot and cold only in the inside of the thumb and outside of the index.

Guttmann, L., said that this was extraordinary, as according to physiological and neurological knowledge the sensibility which recovered first after nerve suture was pain, especially deep pain; therefore he wondered whether the return of touch sensibility was not produced by the function of the adjacent nerves of the median, i.e. ulnar and radial. One must be very careful with conclusions as to recovery of sensibility. There was no doubt that touch sensibility in the autonomous zone of a peripheral nerve definitely recovered much later than pain sensibility. This case should be very carefully studied by a skilled neurologist for detailed sensory tests.

Benassy, $\mathcal{F}$., replied that Dr. Grossiord had looked at the patient too. But in that particular case the sensibility could come from no other pathway since he had cut the median.

Bardot, $A$. (France), said that as there had been a recovery of the proximal muscles would it not be interesting to perform the operation earlier and what delay would Dr. Benassy give? By doing the operation earlier would not a recovery of the thenar muscles at least have a better chance?

Benassy, f., replied that it would be better if surgery could be done much earlier, one month would probably be the best delay. But as they could always hope for recovery they were in between two dangers - operating too soon or waiting too long.

$K e r r, W . G$., wanted to have confirmation what muscles of the arm were lost when the musculo-cutaneous was divided, because function of the arm seemed extremely good.

Benassy, $\mathcal{F}$., replied that the patient had lost the lower head of the biceps. By exercise he developed the long head of the biceps and the brachioradialis, the latter being supplied by the radial nerve.

Guttmann, L., said he was not surprised that the flexion of the forearms was so good because one must remember that the flexors of the fingers were also flexors of the forearms. The same applied to the pronator teres. So the loss of the biceps even would not be too horrible because if there was really good recovery of function of the flexors of the wrist, the flexor carpi radialis, and long flexors of the fingers the flexion of the forearm must be good. 